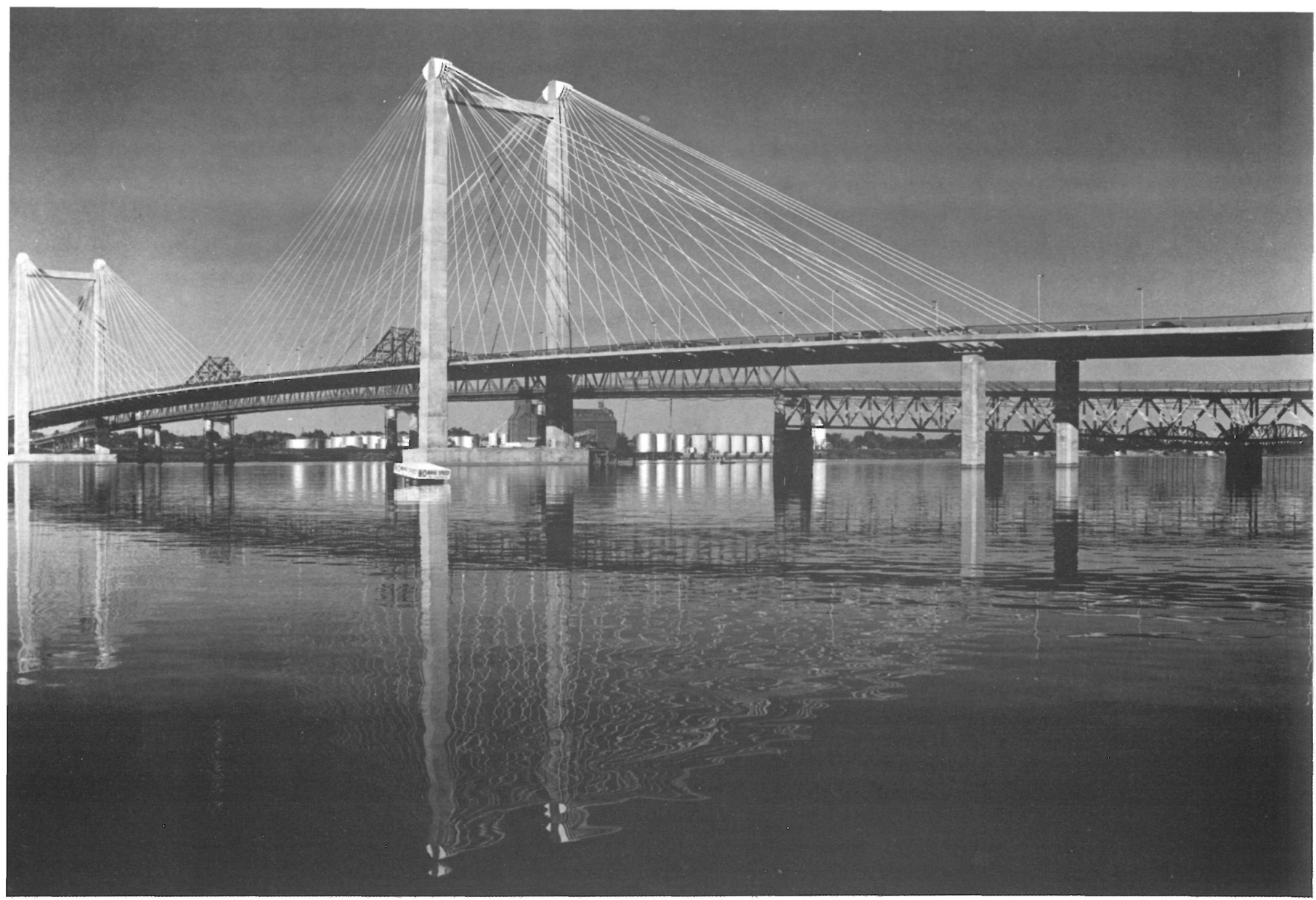

sinopsis El puente Intercity, sobre el río Co-
lumbia en el Estado de Washington, lumbia en el Estado de Washington, es de tipo atirantado, de hormigón pretensado - terminado en 1978 - que
introduce en la ingeniería americana introduce en la ingeniería americana
de puentes un sistema de construcde puentes un sistema de construc-
ción eficiente, duradero y simple. En este artículo se describe la obra, los estudios ingenieriles realizados y las formas y procedimientos de montaje de la estructura.

El puente, de unos $763 \mathrm{~m}$ de longitud, se proyectó con el objetivo especial de conseguir una gran estabilidad de la estructura en cualquier hipótesis de carga, una vida de servicio larga y un bajo coste inicial, así como escaso entretenimiento.

\section{Puente Intercity, sobre el río Columbia}

EE. UU.

ARVID GRANT y asociados, Inc., ingenieros 564-30

El puente Intercity, que reemplazó a otro más estrecho de vigas de acero -50 años más viejo-, cruza el río Columbia y enlaza las ciudades de Pasco y Kennewick, bajo cuyos auspicios y con la asistencia del Departamento Federal de Carreteras se realizó.

Las fechas más importantes del proyecto fueron: en 1972 tuvo lugar la concepción; en 1973 se fijaron los fines; en 1975 se firmó el contrato, y en septiembre de 1978 se abrió al público. Su coste total fue de unos 2.000 millones de pesetas.

El nuevo puente posee $763 \mathrm{~m}$ de longitud, con una anchura de 24,38 m en aceras; dispone de cuatro vías para tráfico. Los tres vanos principales tienen unas longitudes de: 124, 299 y $124 \mathrm{~m}$ respectivamente. Para realizar el dintel se usaron 62 elementos de viga longitudinal de $24,38 \mathrm{~m}$ de anchura, $8,23 \mathrm{~m}$ de longitud y $2,13 \mathrm{~m}$ de espesor, que pesan cada uno cerca de 300 toneladas. 


\section{PLANEAMIENTO}

El Columbia, en la ciudad de Washington, es un río de bastante anchura y profundidad. La puesta en funcionamiento, el año 1954 de la Presa McNary, elevó el nivel del río unos $7,62 \mathrm{~m}$, con lo que hizo más difícil la construcción de un puente en sus zonas de remanso.

Aunque los americanos se han considerado siempre como gente muy ingeniosa y técnicamente muy creativa, esa creatividad en ingeniería de puentes ha estado dormida durante algún tiempo. Las grandes necesidades aumentan la creatividad y agudizan el ingenio, como en este caso particular.

En la búsqueda de la solución idónea para un puente sobre el río Columbia se pasó revista a todos los sistemas conocidos y posibles de construcción con objeto de encontrar uno que tuviera en cuenta todos los factores-geometría del lugar, profundidad del agua, suelos de la cimentación, aspectos hidráulicos del río, las necesidades de los municipios, etc. Entre los factores más destacados se encuentra la navegabilidad del río, lo que requiere espacios libres para los remolcadores, las barcas y el tráfico de recreo.

Entre las distintas soluciones propuestas se aprecian:

- Vanos de vigas de acero de alma llena.

- Puente de hormigón prefabricado in situ.

- Puente de viga de hormigón realizada por lanzamiento.

- Puente atirantado de viga-cajón de acero.

- Puente atirantado de hormigón pretensado construido mediante dovelas.

Se eligió la última solución por las siguientes razones:

a) Gustó la concepción del proyecto y el aspecto de la estructura.

b) Esta solución era la que tenía el mínimo número de grandes pilas en la parte profunda del río.

c) Asimismo, era la de mayor seguridad a pesar de lo escarpado del terreno.

d) La mayor parte de la obra se podía hacer con material local -hormigón- y, por eso, el proyecto estaría menos sujeto a las fluctuaciones del mercado y de los precios de los materiales.

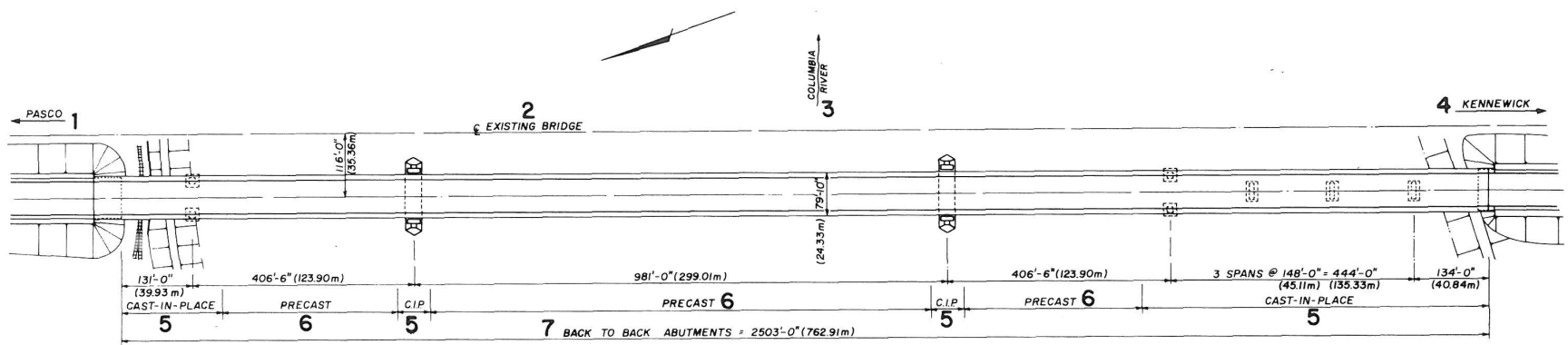

PLANTA

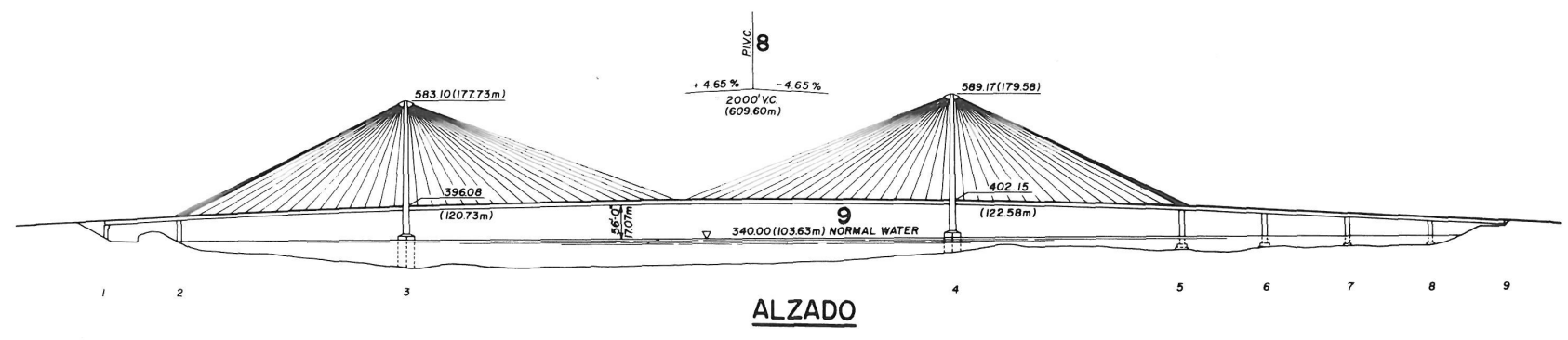

\section{planta y alzado}




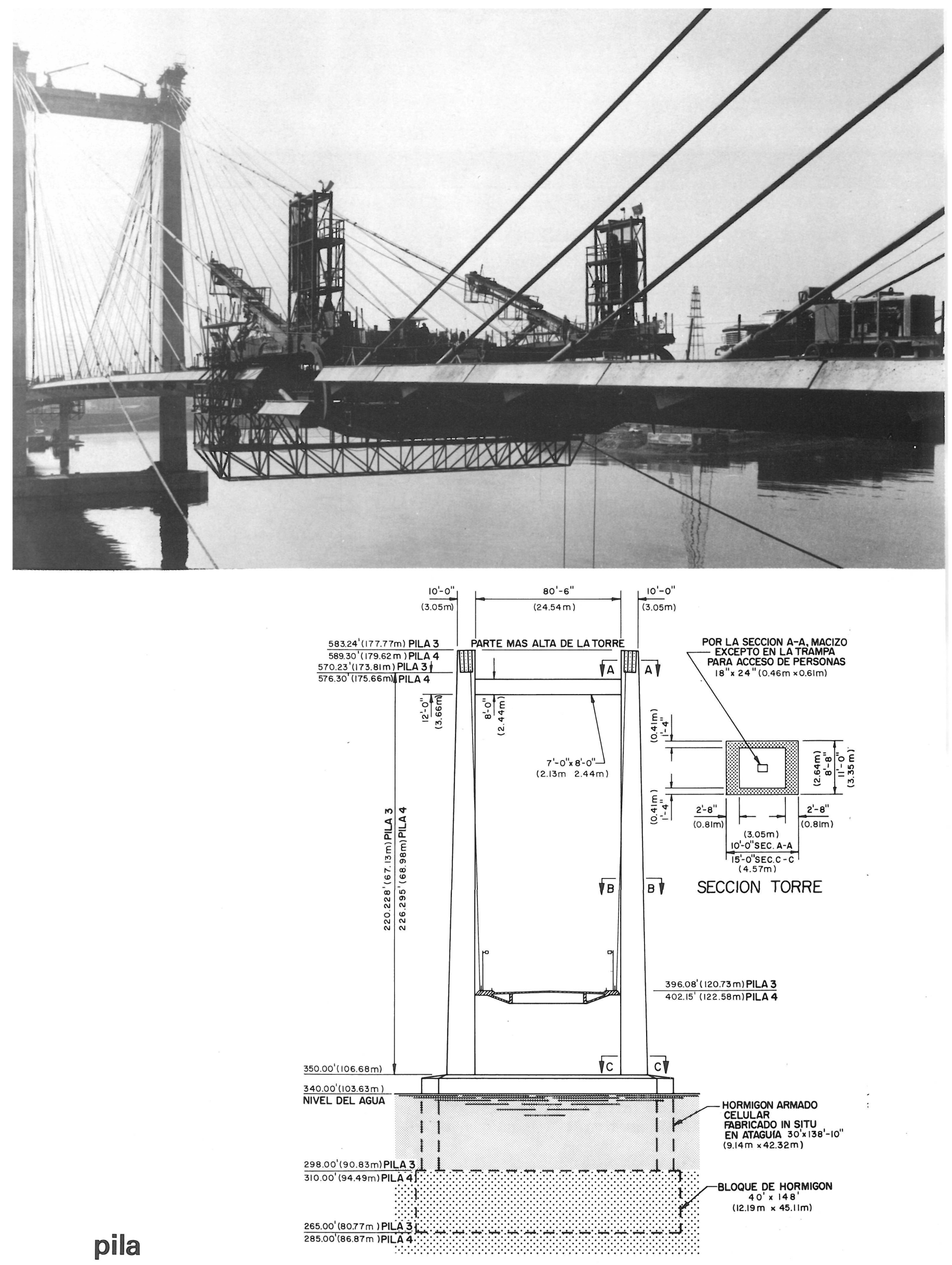




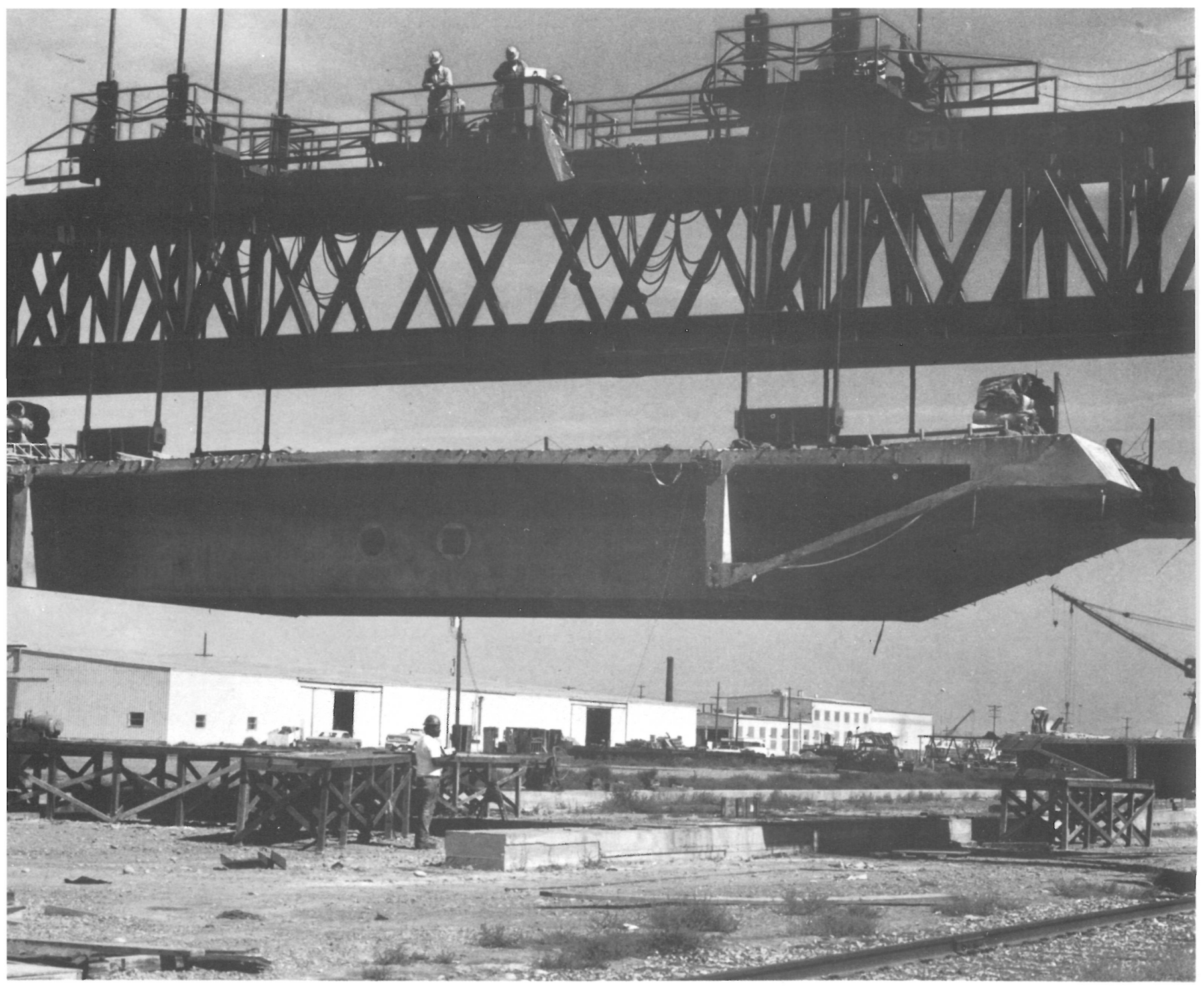

\section{PROYECTO}

Los elementos menos tradicionales del proyecto eran:

a) Una viga delgada sobre muchos soportes dúctiles.

b) Simultáneo comportamiento estructural en los tres planos coordenados.

c) Respuesta dinámica y aerodinámica a las diversas solicitaciones-viento, vibraciones, choques.

d) Interdependencia de los dispositivos y detalles estructurales con los detalles y métodos de montaje y erección.

La estructura del puente consiste en una losa de hormigón apoyada en vigas transversales de $2,74 \mathrm{~m}$ de canto y $8,23 \mathrm{~m}$ de anchura, en cuyos bordes se hacen los anclajes de los tirantes.

Aunque el trabajo analítico, necesario para la verificación y afinamiento de los conceptos y de los detalles del trabajo requirió largos y complicados modelos matemáticos, el resultado final es muy simple en su aspecto y en su acción estructural con muy pequeñas fluctuaciones en las tensiones cualquiera que sean las solicitaciones.

Mientras que todos los componentes estructurales de hormigón y de acero, en carga permanente, sobrepasan el 45 por 100 de su capacidad tensional, las fluctuaciones de las tensiones serán inferiores al 10 por 100 durante toda la vida de servicio de la estructura, lo que hace que la probabilidad de rotura por fatiga del material sea pequeña y que su durabilidad sea bastante grande. 
Los cables son 144, ordenados en dos planos paralelos, sujetos a la parte superior de los pilones mediante dispositivos especiales de acero soldado y anclados en los bordes de la viga de hormigón, con intervalos de 8,23 m. Este nuevo tipo de cable, que se usó por primera vez con esta obra, está hecho de alambres de acero de 6,35 $\mathrm{mm}$ de diámetro, análogos a los usados en el hormigón pretensado, cuando se colocan en línea recta. El número de alambres de cada cable varía de 83 a 280, según cuál sea la situación y la carga del cable. Los alambres fueron revestidos de conductos de polietileno y, una vez instalados, se rellenó el espacio entre los alambres y el conducto mediante una lechada de cemento portland para protegerlo contra la corrosión. Los anclajes son del tipo Hi-Am (gran amplitud), muy desarrollados por Alemania y Suiza con objeto de reducir la probabilidad de fatiga. Los cables se ensayaron, antes de colocarlos, en los laboratorios de la Universidad de Texas no produciéndose pérdidas de resistencia ni fatiga.
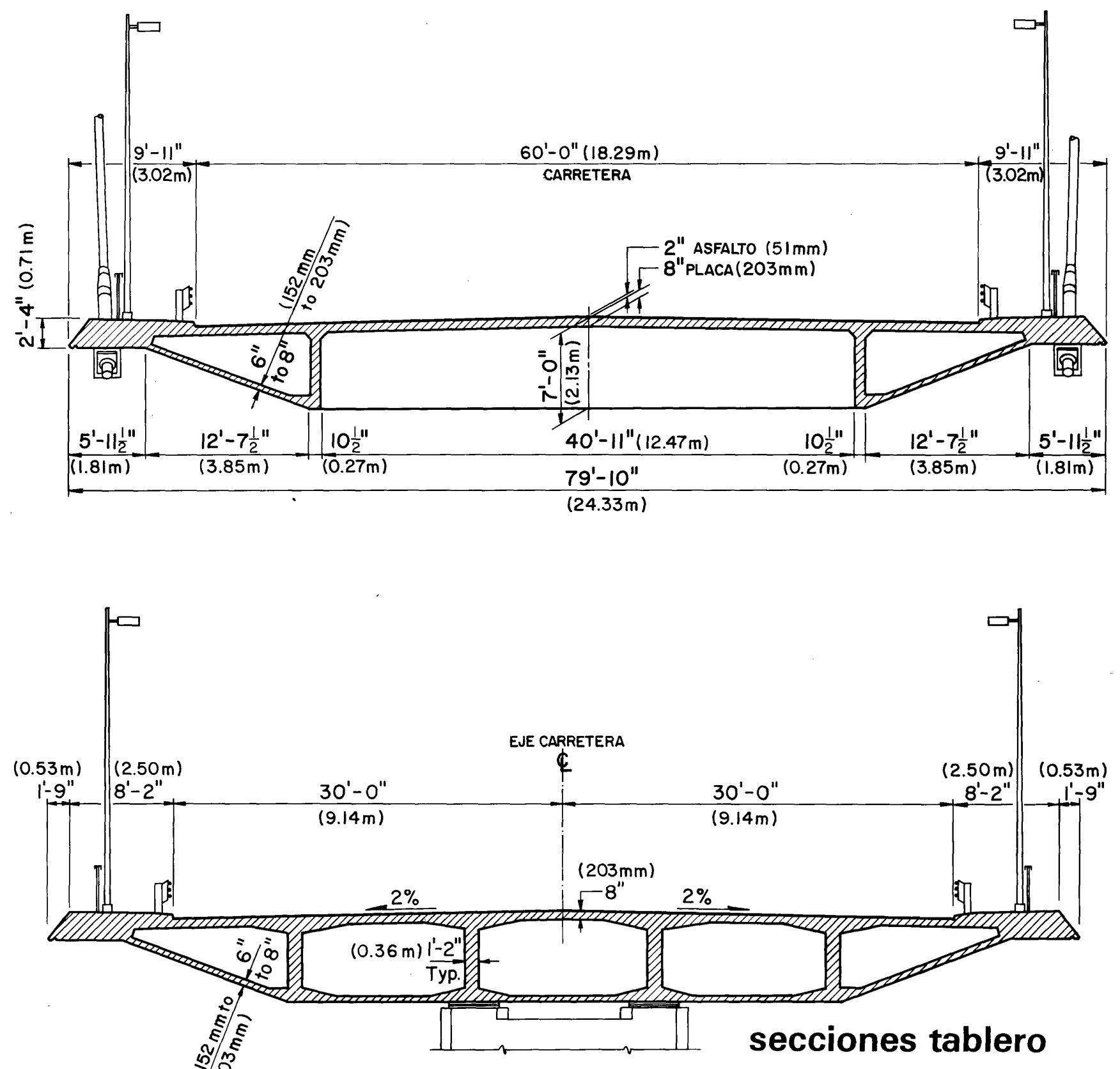
La viga de hormigón distribuye las cargas de los vehículos sobre el sistema sustentante y reduce las fuerzas del viento para que no se produzcan solicitaciones indebidas. Los cables - largos, delgados y duros - sí están sujetos a las vibraciones resultantes de la solicitación del viento; con el fin de minimizarlas se colocan, cerca de los anclajes, amortiguadores duros de neopreno. De esta manera se consiguió que, mientras en un estado parcial los cables se excitan por un viento ligero, en el ustado final no se produzcan vibraciones visibles.

Ei puente tiene una única junta de dilatación para permitir los movimientos debidos a los cambios de temperatura y a otros factores. La viga está libremente apoyada sobre los pilares-torres principales, mientras que está fijada en el estribo norte. Si se produjese una aceleración longitudinal - terremotos u otras causas - mayor que la prevista en el proyecto, las barras verticales de acero del apoyo fijo fallarán por cortante. De esta manera la viga del puente, que en su estado actual tiene una frecuencia de aproximadamente 2 ciclos por segundo, disminuirá su frecuencia a menos de 0,1 ciclos por segundo, con lo que se hará insensible a posteriores aceleraciones.

El puente es insensible a las excitaciones dinámicas debido a la colocación no armónica de los elementos-acero y hormigón. También es un sistema autoamortiguante de algunas solicitaciones.

\section{PROCESO CONSTRUCTIVO}

Según sea el tipo de puente hay un método de construcción tradicionalmente usado y suficientemente probado pero, sin embargo, para realizar el puente Intercity no existía tradición en qué apoyarse.

El proceso de construcción consistió: en la prefabricación de las dovelas del puente, la colocación de dos grandes estructuras de elevación en los segmentos iniciales de la viga sujetándolas con cables provisionales desde la parte superior del pilar, la elevación in situ de las dovelas, la colocación del acero de ferralla y el de pretensado. Después se cubrian las superficies de contacto con resina epoxy, se conectaban las armaduras a la dovela -anteriormente colocada - pretensando la nueva dovela junto a la anterior y transfiriendo entonces toda la carga, con la ayuda de cables provisionales, a los cables permanentes del puente (dos por cada dovela). Este proceso requiere grandes transferencias de tensiones junto con sus correspondientes cambios dimensionales, por lo que se estudió con mucho detalle buscando la máxima precisión.

Las dovelas se moldearon en una zona próxima situada a la orilla del Río Columbia. Este moldeo se hizo siguiendo un plan ya determinado. Cada elemento se moldeó junto a los ya previamente moldeados, con objeto de obtener superficies de contacto que encajaran perfectamente. Para reducir los cambios dimensionales, debidos a la retracción y a la fluencia, los elementos premoldeados se almacenaron y curaron durante seis meses.

El montaje de la viga requirió cerca de seis meses, terminándose tal como se había previsto $\sin$ necesidad de procesos de corrección de geometría y sin producirse accidentes.

\section{DIRECCION Y ADMINISTRACION DEL PROYECTO}

Las grandes obras públicas, como este puente, requieren mucho tiempo, como mínimo 12 años o más desde la iniciación hasta el acabado, consumiéndose la mitad de este tiempo en la resolución de múltiples procedimientos administrativos.

Para ayudar a resolver estas dificultades, los dos municipios crearon un comité compuesto por los alcaldes de las dos ciudades y un presidente elegido por ellos. Este comité, en colaboración con el ingeniero director de la obra, realizó ésta de un modo rápido y dentro del presupuesto.

\section{ESTETICA Y ACOGIDA PUBLICA}

Para que un proyecto sea bueno debe ser simple; de hecho, un proyecto no es completo si contiene elementos que no son necesarios. 
El espectador, que no conoce los razonamientos, el análisis y las decisiones que ha habido que tomar antes de la terminación de un proyecto debe, al ver una obra acabada, notar una sensación de orden y de lógica para pensar que es estéticamente aceptable. Esto se consiguió en el Puente Intercity.

El antiguo puente tenía un tráfico de unos 14.000 vehículos/día. El nuevo puente, que ya durante la primera semana alcanzó un tráfico de 19.000 vehículos/día, dos meses más tarde tenía un tráfico de 20.000 vehículos/día.

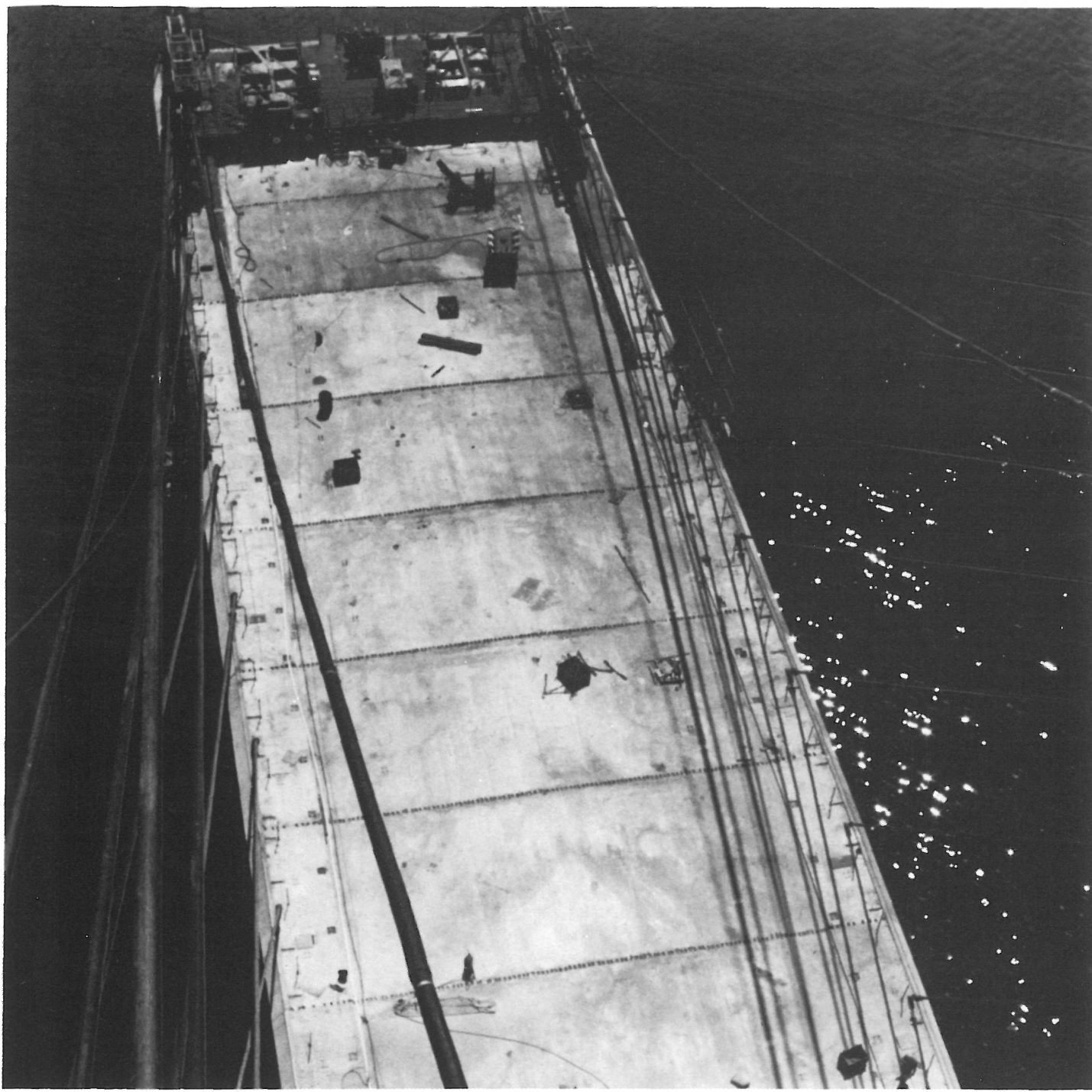

\section{résumé}

LE PONT INTERCITY; SUR LE

COLUMBIA - Etats Unis

Arvid Grant et associés, ingénieurs

Le pont Intercity, sur le Columbia, dans l'état de Washington, est du type haubané, de béton précontraint - terminé en 1978 . et introduit dans le génie civil américain des ponts, un système de construction efficien durable et simple. Cet ouvrage est décrit dans cet article ainsi que les études réalisées et les systemes et procédés de montage de la structure.

Ce pont, de $763 \mathrm{~m}$ de long. a été projeté

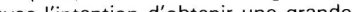
sous nimpore quelle hypothese de charge, une lovition revient initial bas ainsi qu'un minimun d'en-

\section{summary}

INTERCITY BRIDGE OVER

THE COLUMBIA RIVER, U.S.A

Grant and Associates, Engineers

The Intercity Bridge over the Columbia River in the State of Washington, completed in 1978, was built in prestressed concrete using the traction method and introduces in the American bridge engineering an efficient, lasting and simple construction system. This article describes the job the engineering studies carried out and the ways and means used to erect the structure.

The $763 \mathrm{~m}$ long bridge was specially designed to achieve a high structural stability under to achieve a high structural stability under any concleivable load, a long service life maintenance.

\section{zusammenfassung}

INTERCITY-BRUCKE UBER DEM COLUMBIA-FLUSS - USA

Arvid Grant und Partner, Ingenieure

Die über dem Columbia-Fluss im Staate Washington nach Zugverstrebungsbauart mit vorgespannten Beton errichtete -im Jahre 1978 fertiggestellte- Intercity Brücke bedentete die Einführung cines wirksamen dentete die Einfuhrung eines wirksamen, dauerhaften und einfachen Bausystems in das amerikanische Brückeningenieurwesen. In diesem Artikel werden das Bauwerk, die durchgeführten Ingenieurarbeiten und die Struckturverfahren beschrieben.

Die Brücke von ca. $763 \mathrm{~m}$ Länge wurde derart ausgelegt, dass eine grosse Stabilität der Struktur bei irgendwelcher Belastung, eine lange Lebensdauer und geringe Bau-
und Unterhaltskosten gegeben sind. 